\title{
Conocimiento matemático de profesores de primaria en formación para la enseñanza de la probabilidad: un estudio exploratorio
}

\author{
Prospective primary school teachers' mathematical \\ knowledge for teaching probability: an exploratory \\ study
}

\section{Conhecimento matemático de professores dos anos iniciais do Ensino Fundamental em formação para o ensino de probabilidade: um estudo exploratório}

\author{
Carmen Batanero Bernabeu* \\ Emilse Gómez Torres" \\ José Miguel Contreras García" \\ Carmen Díaz Batanero ${ }^{* * * *}$
}

\begin{abstract}
Resumen: En este trabajo se evalúan algunos elementos del conocimiento matemático común y especializado del contenido y del conocimiento del contenido y los estudiantes en relación con la probabilidad elemental en una muestra de profesores en formación. El conocimiento común del contenido se evalúa a partir de las soluciones de 157 profesores en formación a tres problemas. Una vez discutidas con los participantes estas soluciones, se analizan dos componentes del conocimiento de 81 de ellos, trabajando en pequeños grupos: (a) El conocimiento especializado del contenido se evalúa a partir de los contenidos matemáticos identificados en los problemas; (b) el conocimiento del contenido y los estudiantes a partir de la identificación y justificación de respuestas de alumnos de educación primaria. Aunque en la evaluación inicial se observó un bajo razonamiento probabilístico, en la segunda los participantes son capaces de identificar y explicar las respuestas incorrectas de los alumnos.
\end{abstract}

Palabras clave: Conocimiento Matemático para la Enseñanza. Probabilidad. Educación Primaria.

\footnotetext{
*Catedrática de Didáctica de las Matemáticas, Universidad de Granada. E-mail: < batanero@ugr.es>

** Profesora Asistente del Departamento de Estadística, Universidad Nacional de Colombia. E-mail: <egomezt@unal.edu.co>

*** Profesor ayudante doctor del Departamento Didáctica de las Matemáticas, Universidad de Granada. E-mail: <jmcontreras@ugr.es>

${ }^{* * * *}$ Profesora contratada doctor del Departamento de Psicología, Universidad de Huelva, España. E-mail: <carmen.diaz@dpsi.uhu.es>
} 


\begin{abstract}
This study assesses some elements of common and specialized mathematical knowledge and of knowledge of content and students in relation to elementary probability from a sample of prospective primary school teachers. Common knowledge of content is assessed from the individual solution of 157 prospective teachers to three problems. Once the solution to these problems was discussed with the participants, two additional components of these teachers' mathematical knowledge for teaching were analyzed based on small group work developed by 81 participants: (1) To assess specialized knowledge of content we asked participants to identify the tasks mathematical content; (b) to assess their knowledge of content and students we asked them to identify and justify correct and incorrect answers to the problems given by primary school children. While in the initial assessment we observed a poor probabilistic reasoning, in the second part they were able to identify and explain incorrect answers given by students.
\end{abstract}

Keywords: Mathematical Knowledge for Teaching. Probability. Primary School.

Resumo: Neste trabalho foi realizada uma avaliação de alguns elementos do conhecimento matemático comum e especializado e do conhecimento do conteúdo dos alunos sobre a probabilidade elementar de professores do ensino primário em formação. O conhecimento comum do conteúdo é avaliado a partir das soluções de 157 professores em formação a três problemas. Uma vez que os participantes discutiram com estas soluções, analisamos dois componentes do conhecimento matemático para o ensino de 81 desses alunos, trabalhando em pequenos grupos: a) O conhecimento especializado do conteúdo é avaliado com base no conteúdo matemático dos problemas identificados, (b) o conhecimento do conteúdo e os alunos são avaliados com base na identificação e justificação das respostas de alunos do ensino fundamental. Enquanto na avaliação inicial mostrou um raciocínio probabilístico pobre, na segunda, os participantes são capazes de identificar e explicar as respostas incorretas dos alunos.

Palavras-chave: Conhecimento matemático para o ensino. Probabilidade. Ensino Fundamental.

\title{
Problema de investigación
}

Actualmente la probabilidad se enseña en España todos los ciclos de la Educación Primaria (MEC, 2006) con el objetivo de proporcionar a los niños una experiencia estocástica desde su infancia, reforzando sus intuiciones probabilísticas. Se sigue la tendencia iniciada en el currículo americano (NCTM, 2000; CCSI, 2010) y mediante un lenguaje sencillo, juegos, experimentos y observación de fenómenos naturales, se intenta que el niño aprenda a reconocer la aleatoriedad y que al final de esta etapa educativa, sea capaz de comparar y estimar algunas probabilidades sencillas. 
Para llevar a cabo esta enseñanza con éxito, se requiere una gama muy variada de conocimientos y experiencia por parte del profesor de matemáticas. El objetivo de este trabajo fue evaluar algunos elementos del conocimiento común y especializado del contenido y del conocimiento del contenido y los estudiantes en relación con la probabilidad elemental en una muestra de profesores en formación.

\section{Marco teórico}

La descripción hecha por Shulman (1986) del conocimiento pedagógico del contenido, como un conocimiento profesional específico del profesor, promovió un gran número de investigaciones orientadas a la evaluación y desarrollo del mismo en los profesores de matemática (e.g.: LLINARES; KRAINER, 2006; WOOD, 2008; EVEN; BALL, 2009).

Nuestro trabajo se apoya en el marco propuesto por Hill, Ball y Schilling (2008), quienes denominan Conocimiento Matemático para la Enseñanza (MKT) al "conocimiento matemático que utiliza el profesor en el aulapara producir instrucción y crecimiento en el alumno" (HILL; BALL; SCHILLING, 2008, p. 374), que está conformado por el conocimiento del contenido y el conocimiento pedagógico del contenido. Dentro del primero se distinguen tres componentes: El Conocimiento Común del Contenido (CCK), es el que posee cualquier persona instruida en matemática; el Conocimiento Especializado del Contenido (SCK), aplicado por el profesor para articular tareas de enseñanza (por ejemplo, buscar actividades para presentar el concepto de independencia); y finalmente el Conocimiento en el Horizonte Matemático, es una visión más amplia de la matemática que la requerida para la enseñanza (sirve al profesor para establecer conexiones de un contenido particular con otros temas anteriores o posteriores) (BALL; THAMES; PHELPS, 2008). El conocimiento pedagógico del contenido se desglosa en tres componentes: El Conocimiento del Contenido y los Estudiantes (KCS) incluye el conocimiento de sesgos, errores y dificultades, estrategias y aprendizaje del alumno; el Conocimiento del Contenido y la Enseñanza que incluye aquellos aspectos relacionados con los procesos adecuados para enseñar y evaluar un tema; y el último componente es el Conocimiento del Currículo.

La finalidad de este trabajo es evaluar algunos elementos del conocimiento común y especializado del contenido y del conocimiento del contenido y los estudiantes en relación con la probabilidad elemental en una muestra de profesores en formación. A continuación presentamos los antecedentes del trabajo y describimos su metodología. En segundo lugar, analizamos las soluciones dadas por 157 estudiantes para profesor de Educación Primaria a tres problemas abiertos, para evaluar su conocimiento común del contenido. Seguidamente se evalúa el conocimiento especializado del contenido, a partir de los contenidos matemáticos 
que 81 de estos participantes, trabajando en grupo, identifican, en dichos problemas, después de haberse realizado una discusión colectiva de las soluciones a los problemas y actividades de simulación. El conocimiento del contenido y los estudiantes se deduce de las evaluaciones que los mismos grupos de participantes realizan de las respuestas al problema proporcionadas por algunos niños en investigaciones previas. Finalizamos con la discusión y conclusiones.

\section{Antecedentes}

A pesar del gran esfuerzo de investigación sobre formación de profesores en los últimos años, son pocos los trabajos sobre conocimiento matemático del profesor para enseñar probabilidad. En lo que sigue, sintetizamos los trabajos centrados en los conocimientos del contenido por parte de futuros profesores de Educación Primaria y los que tratan el conocimiento pedagógico del contenido por parte de profesores de Educación Primaria en ejercicio o en formación.

\section{Conocimiento de la probabilidad}

Las escasas investigaciones sobre conocimiento probabilístico de los profesores o futuros profesores indican carencias formativas. Entre ellos, Azcárate (1995) pasó un cuestionario a 57 futuros profesores de Educación Primaria, encontrando que pocos mostraban una idea clara de la aleatoriedad, y detectando falta de esquemas combinatorios y dificultades en cálculo de probabilidades y en diferenciar los juegos equitativos y no equitativos. Serrano (1996) observó, en entrevistas a 10 profesores en formación, dificultades con el concepto de independencia y el sesgo de equiprobabilidad (LECOUTRE, 1992), que consiste en creer que todos los sucesos asociados a un experimento aleatorio son equiprobables.

Resultados similares fueron también encontrados por Batanero, Cañizares y Godino (2005) en un estudio con 132 profesores de Educación Primaria en formación, y por Batanero, Arteaga, Serrano y Ruiz (2014) en otro con 200 profesores en formación del mismo nivel educativo.

Contreras (2011) analiza las respuestas de 183 estudiantes para profesor de Educación Primaria a tres tareas, para evaluar su conocimiento sobre el cálculo de probabilidades en tablas de contingencia $2 \times 2$. Aunque la mayoría calculó correctamente probabilidades simples, sólo el 41\% fue capaz de calcular la probabilidad compuesta y 44\% la condicional. Mohamed (2012) analizó las respuestas de 102 profesores de Educación Primaria en formación a otro cuestionario, observando que la mayoría calculó correctamente la probabilidad simple, estimó correctamente la probabilidad a partir de la frecuencia relativa, y 
reconoció un juego no equitativo en un experimento simple; sin embargo, pocos resolvieron correctamente problemas sobre variable aleatoria y muestreo.

Prodromou (2012) investigó las conexiones que un grupo de 100 profesores de Educación Primaria en formación establecen entre el enfoque clásico y frecuencial de la probabilidad en tareas de lanzamiento de dados. Los sujetos mostraron confusión sobre la necesidad de tener en cuenta el orden de los dados al estudiar la probabilidad de su suma y no establecieron relaciones entre las probabilidades teóricas y las frecuencias relativas del experimento, por no comprender bien la idea de convergencia.

Smith y Hjalmarson (2013) analizaron la compresión de la aleatoriedad, de 45 profesores de Educación Primaria en formación, a lo largo de un curso de estadística. Al comienzo del curso, algunos profesores no ligaron las ideas de aleatoriedad y convergencia; posteriormente, las actividades les ayudaron a mejorar sus intuiciones previas.

\section{Conocimiento pedagógico sobre la probabilidad}

Son más escasos los trabajos centrados en este componente del conocimiento del profesor. Entre ellos, Watson (2001) realiza un estudio con 28 profesores de secundaria, de los cuales solo 13 reconocieron las dificultades específicas de sus alumnos en el trabajo con la probabilidad, pues el resto se centró en aspectos de procedimiento, y la mayoría tuvo limitaciones al valorar algunas respuestas conceptuales.

Stohl (2005) examinó la interpretación de 35 profesores de educación secundaria acerca del desempeño de algunos estudiantes cuando desarrollaban una tarea de simulación. Los profesores se limitaron a criticar la ausencia de ideas formales sobre probabilidad en sus alumnos, ignorando el desarrollo de ideas probabilísticas intuitivas reflejadas en las acciones de los niños con el software y su uso apropiado del lenguaje probabilístico.

Carter (2008) pidió a 210 futuros profesores de secundaria evaluar la solución de un estudiante ficticio y explicar su razonamiento en tres problemas sobre independencia y probabilidad conjunta, siendo muy pocos los que supieron dar una explicación aceptable de los razonamientos. Mohamed (2012) propone una tarea similar (evaluar y explicar el razonamiento de alumnos ficticios) en cuatro problemas de probabilidad, resueltos previamente, a 102 futuros profesores de Educación Primaria, trabajando en pequeños grupos (2-3 sujetos por grupo). Aunque la mayoría de los grupos discrimina bien las respuestas correctas e incorrectas; no siempre logran explicar las causas de error y en ocasiones su respuesta previa al problema es inconsistente con la evaluación del estudiante 
ficticio. Dificultades similares son encontradas por Ortiz, Batanero y Contreras (2012) en una muestra de 167 profesores en formación al evaluar las respuestas de niños sobre juegos equitativos, trabajando en pequeños grupos.

Nuestra investigación completa las anteriores, considerando nuevas tareas, y modificando las condiciones de la evaluación, tratando de desarrollar, a la vez que se evalúan, los componentes citados del conocimiento matemático para la enseñanza de la probabilidad en los participantes. Para ello se presenta la evaluación como parte de un ciclo formativo en que, realizada la evaluación inicial de su conocimiento matemático común, se promueve una discusión de las respuestas de los participantes, complementada con actividades de simulación. Con ello tratamos de reforzar su conocimiento matemático común de la probabilidad antes de llevar a cabo la evaluación del conocimiento especializado y el conocimiento del contenido y el estudiante. En lo que sigue se presentan el método y los resultados.

\section{Método}

\section{Participantes}

En la primera parte de la evaluación participaron 157 profesores de en formación de la Universidad de Granada, alumnos de segundo año del Grado de Maestro en Educación Primaria. Estos alumnos habían estudiado probabilidad simple y condicional, durante la educación secundaria y en el curso de "Bases matemáticas para la Educación Primaria" en primer año de estudios. Parte de la muestra (81 sujetos) participó también en la segunda parte del estudio, trabajando en pequeños grupos (39 parejas y un grupo de tres).

\section{Instrumento}

El cuestionario usado en esta investigación consta de dos partes. En la primera (Figura 1) se pide a los participantes resolver tres problemas para evaluar su conocimiento común del contenido. El ítem 1, tomado de Serrano (1996), evalúa el sesgo de equiprobabilidad (LECOUTRE, 1992), que se muestra cuando el alumno da la respuesta (d); siendo la respuesta correcta la alternativa (a). El ítem 2, adaptado de Azcárate (1995), evalúa la comprensión de juego equitativo y la capacidad para estimar la ganancia requerida para convertirlo en equitativo. La respuesta correcta es que el juego no es equitativo y que Luis debe ganar 3 euros pues su probabilidad es la tercera parte de la de Miguel. El ítem 3, adaptado de una tarea propuesta por Falk y Wilkening (1998) a niños entre 6 y 14 años, evalúa la capacidad de comparación de probabilidades. Hay dos respuestas correctas: 4 bolas blancas y 6 negras en cada caja o 2 bolas blancas y 3 negras en una caja y 6 bolas blancas y 9 negras en la otra. 
Figura 1 - Parte 1 del cuestionario

\section{Parte 1. Resuelve los siguientes problemas, explicando con detalle tu res- puesta}

Ítem 1. ¿Cuál de estos resultados es más fácil que ocurra al lanzar tres veces un dado? Marca la respuesta correcta:

a.Obtener un 5 , un 2 y un 3

b.Obtener dos veces un 5 y una vez el 3

c.Obtener tres veces el 5

d.Todos estos resultados son igualmente probables

¿Es alguno de estos resultados menos probable que los otros dos? ¿Cuál o cuáles?

Ítem 2. Miguel y Luis juegan a un juego con dos dados ordinarios (como sabes cada dado está numerado del 1 al 6). Tiran los dos dados y multiplican los números obtenidos. Miguel gana un euro si el producto es par; si el producto es impar, Luis gana un euro

¿Te parece que el juego es equitativo? ¿Por qué? ¿Cuánto tendría que ganar Luis, si Miguel gana 1 euro, para que el juego sea equitativo?

Ítem 3. Pablo tiene 5 bolas blancas y 7 negras en una caja. Miguel tiene otra caja con 3 bolas blancas y 5 negras. ¿Cuántas bolas, negras o blancas, pasarías de una caja a la otra para que ambos niños tengan la misma probabilidad de extraer una bola negra?

Fuente: Desarrollado por los autores.

La segunda parte del cuestionario (Figura 2) se compone de tres preguntas, que tienen en cuenta el conocimiento matemático utilizado por el profesor en la resolución de problemas profesionales, como puede ser el análisis de los problemas, identificando lo matemáticamente relevante en la situación o bien evaluando a los estudiantes (LLINARES, 2009). Se sigue también la metodología propuesta por Godino (2009).

Siguiendo las sugerencias de este autor, la pregunta 1, orientada a evaluar el conocimiento especializado del contenido, pide analizar el contenido matemático necesario para resolver cada ítem, que incluye los conceptos de aleatoriedad, experimento aleatorio y suceso, casos favorables y posibles, probabilidad clásica y regla de Laplace. En los ítems 1 y 2, además, experimento y suceso compuesto; en el ítem 2, también, variable aleatoria, juego equitativo y esperanza matemática. Finalmente se utilizarían conceptos numéricos (número, proporción) junto con sus propiedades. Como procedimientos, los alumnos necesitarían enumeración sistemática, comparar y calcular probabilidades, y procedimientos aritméticos, como suma u otras operaciones.

Las preguntas 2 y 3 de esta parte del cuestionario estuvieron orientadas a evaluar algunos elementos del conocimiento del contenido y los estudiantes 
en relación con la probabilidad elemental en una muestra de profesores en formación. En el ítem 1, esperamos, que se identifique la respuesta correcta A5. La respuesta A4 es parcialmente correcta, pues el alumno identifica el suceso menos probable, pero no el más probable. El resto de respuestas son incorrectas: A1, porque, aunque en un juego de azar no se puede predecir el resultado exacto, si se puede decir cuál es más probable; A2, porque, aunque identifica el suceso menos probable, no identifica el más probable; A3 realiza una enumeración de casos para la primera pregunta y no distingue el suceso menos probable.

Figura 2 - Parte 2 del cuestionario

Parte 2. Para cada uno de los tres ítems resueltos en la parte 1, mostramos algunas respuestas dadas por alumnos de Educación Primaria.

1. Indica el contenido matemático que tienen que usar los alumnos para dar la respuesta correcta

2. Señala las respuestas correctas e incorrectas

3. Para cada una de las respuestas incorrectas explica cuáles son las posibles intuiciones o estrategias incorrectas que han llevado a los participantes a dar una respuesta errónea

\section{Respuestas de alumnos ficticios al ítem 1}

A1. Todos son igualmente probables; es un juego de azar, no se puede predecir.

A2. Es más difícil obtener tres veces el 5; los otros dos resultados tienen la misma probabilidad.

A3. Es más fácil obtener un 5, un 2 y un 3, porque tienes tres casos diferentes: 523, 325 y 532.

A4. Es más difícil obtener tres veces el 5; para los otros resultados tienes más casos y para este solo 1.

A5. Es más difícil obtener tres veces el 5, porque sólo tienes un resultado; para dos veces el 5 y una el tres tienes tres resultados $(553,535,355)$; para el 5, 2, 3 tienes 6 resultados $(523,532,325,352$, $235,253)$.

\section{Respuestas de alumnos ficticios al ítem 2}

B1. Miguel tiene 2 posibilidades de ganar más que Luis; considero que sea justo que Luis gane 2 euros.

B2. Luis debe ganar 6 euros para que sea justo porque tiene menos posibilidades.

B3. Si a Luis le diesen 3 euros estaría equilibrado, porque por cada vez que le toque a Miguel le dan un euro; pero sólo si le dan a Luis tres oportunidades, ya que Miguel tiene tres veces más oportunidades de ganar.

B4. El juego no es justo porque Miguel tiene muchas más oportunidades; aunque le des más dinero a Luis cuando gane, sigue sin ser justo, porque tiene menos posibilidades de ganar.

B5. Para que el juego sea justo Luis tiene que ganar 3 euros.

\section{Respuestas de alumnos ficticios al ítem 3}

C1. Se puede añadir a la caja de Miguel 2 bolas blancas y 2 negras sin cambiar la de Pablo.

C2. Se puede pasar 1 bola blanca de la caja de Pablo a la de Miguel.

C3. Hay en total 8 bolas blancas y 12 negras entre las dos cajas. Las bolas están en razón 8/12, es decir 2 blancas por cada 3 negras. Si dejamos en la caja de Pablo 2 blancas y 3 negras, tenemos que pasar 3 blancas y 4 negras a la de Miguel; entonces Miguel tendrá 6 blancas y 9 negras; sus bolas también están en razón $6 / 9=2 / 3$ y tienen la misma probabilidad.

C4. Pasando 1 bola blanca y 1 negra de la caja de Miguel a la de Pablo.

Fuente: Desarrollado por los autores. 
En el ítem 2 el alumno B5 da una solución correcta; mostrando idea clara del juego equitativo y buen razonamiento proporcional. La respuesta de B3 es parcialmente correcta, pues calcula correctamente las probabilidades y el premio de Luis, pero añade una condición innecesaria, que fue dada por algunos niños en las entrevistas realizadas en la investigación de Cañizares (1997). Son incorrectas el resto de respuestas: B1 ha estimado bien la razón de posibilidades a favor de cada jugador, pero las compara en forma aditiva y establece un premio incorrecto; B2 tiene una buena percepción de la ausencia de equiprobabilidad, pero falla al establecer el valor del premio; B4 muestra poco razonamiento combinatorio (no es capaz de calcular el número de casos favorables), tampoco comprende la idea de juego equitativo; piensa que aunque se cambie la cantidad de dinero que gana cada jugador seguiría sin ser equitativo, creencia que apareció en niños en la investigación de Cañizares (1997).

En el ítem 3 la respuesta del alumno C3 es correcta; pues ha calculado la proporción total en la suma de bolas blancas y bolas negras en las dos cajas y propone dos urnas con diferente composición, que respeta esta proporción. Son incorrectas el resto de respuestas: $\mathrm{C} 1$, porque, aunque propone dos cajas exactamente iguales, el enunciado pide no variar el número total de bolas; C2 solo tiene en cuenta los casos desfavorables y estaría en la segunda etapa de razonamiento probabilístico, en la teoría de Piaget e Inhelder (1951); C4 ha confundido el nombre de los niños, pues si hiciese el cambio propuesto pasando las bolas de Pablo a Miguel la solución sería correcta.

\section{Procedimiento de recogida de datos}

Los datos se recogieron dentro de una asignatura obligatoria, "Enseñanza y Aprendizaje de la Matemática", a lo largo de tres sesiones de clase. En la primera sesión, los profesores en formación resolvieron individualmente por escrito la primera parte del cuestionario, presentada en la Figura 1. En la segunda sesión (una semana después), y una vez que el investigador recogió y analizó los datos de la primera sesión, se corrigieron colectivamente las respuestas, adoptando el siguiente esquema:

1. El formador de profesores presenta cada uno de los cuatro ítems de la primera parte cuestionario (Figura 1) y pide a los participantes explicar las respuestas dadas por escrito en la primera sesión.

2. Se abre un debate donde los participantes presentan sus respuestas y tratan de diferenciar las correctas e incorrectas. Se realizan actividades de simulación con material manipulativo y con applets representando las situaciones propuestas: lanzamientos de tres dados (ítem 1), producto de los puntos al lanzar dos dados (ítem 2), extracción de 
bolas en urnas (ítem 3). Estas actividades, junto con la enumeración del espacio muestral en cada experimento, facilitan el reconocimiento de soluciones incorrectas en la parte primera.

3. En la tercera sesión (la siguiente semana), los estudiantes resuelven por escrito la parte segunda del cuestionario, trabajando en pequeños grupos. En este artículo se analizan únicamente los datos de 40 grupos (39 parejas y un grupo de tres alumnos; 81 estudiantes en total). En una cuarta sesión se corrigieron colectivamente estas respuestas, adoptando un esquema similar al descrito anteriormente. Se informó a los participantes de la finalidad del estudio y ellos colaboraron voluntariamente.

\section{Resultados}

\section{Resultados sobre el Conocimiento Común del Contenido}

Recogidas las respuestas a la Parte 1 del cuestionario, se realizó un análisis de contenido de las mismas. Debido a la limitación de espacio, presentaremos sólo un resumen de los resultados en las Tablas 1 a 3, que muestran un insuficiente conocimiento del contenido de probabilidad elemental en los profesores en formación.

Tabla 1 - Frecuencia y porcentaje (respecto a columna) de futuros profesores al ítem 1

\begin{tabular}{|c|c|c|c|c|c|c|c|c|}
\hline \multirow{3}{*}{$\begin{array}{l}\text { Menos probable } \\
\text { (Segunda parte) }\end{array}$} & \multicolumn{6}{|c|}{ Más probable (Primera parte) } & & \\
\hline & \multicolumn{2}{|c|}{$\begin{array}{c}\text { Un } 5 \text {, un } 2 \text {, un } 3 \\
\text { (Correcta) }\end{array}$} & \multicolumn{2}{|c|}{ Equiprobable } & \multicolumn{2}{|c|}{ Otra respuesta } & & \\
\hline & Frecuencia & $\%$ & Frecuencia & $\%$ & Frecuencia & $\%$ & Total & $\%$ \\
\hline Tres 5 (Correcta) & 43 & 86,0 & 19 & 18,3 & & & 62 & 39,5 \\
\hline Equiprobable & 1 & 2,0 & 77 & 74,0 & & & 78 & 49,7 \\
\hline Otra respuesta & 4 & 8,0 & 1 & 1,0 & 1 & 33,3 & 6 & 4,8 \\
\hline En blanco & 2 & 4,0 & 7 & 6,7 & 2 & 66,6 & 11 & 7 \\
\hline Total & 50 & & 104 & & 3 & & 157 & \\
\hline
\end{tabular}

Fuente: Datos de la investigación.

En el primer ítem (Tabla 1) solo 50 participantes (31,8\% de la muestra) da la respuesta correcta a la primera parte y 43 (27,3\% de la muestra) dan las dos respuestas correctas; $104(66,2 \%)$ sugieren equiprobabilidad de resultados en la primera parte y $77(49 \%)$ en los dos apartados.

En el ítem 2 (Tabla 2), aunque la mayoría de los participantes identifica correctamente que el juego no es equitativo, sólo algo más de la mitad es además capaz de hallar el premio que lo convierte en equitativo: En general el 
fallo se produjo por un cálculo incorrecto de la probabilidad de cada jugador, debido a dificultad en la enumeración del espacio muestral; en otros casos, se debe a dificultad con la idea de esperanza matemática o fallo en razonamiento proporcional.

En el ítem 3 (Tabla 3), una mayoría da una respuesta correcta, aunque los participantes simplemente distribuyen las bolas blancas y negras en dos cajas con idéntica composición; siendo muy pocos los que dan una composición diferente, pero proporcional, de las dos urnas. Además un 23,4\% no son capaces de resolver el problema.

Tabla 2 - Frecuencia y porcentaje (respecto a columna) de futuros profesores al ítem 2

\begin{tabular}{l|c|c|c|c|c|c}
\hline \multirow{2}{*}{ Respuesta segunda parte } & \multicolumn{3}{|c|}{ Respuesta primera parte } & \multicolumn{2}{c}{} \\
\cline { 2 - 8 } & \multicolumn{2}{|c|}{ No (Correcta) } & \multicolumn{2}{c}{ Sín } & \multicolumn{2}{c}{} \\
\cline { 2 - 8 } & Frecuencia & $\mathbf{0}$ & Frecuencia & $\mathbf{\%}$ & Total & $\mathbf{\%}$ \\
\hline Correcta (3 €) & 77 & 54,6 & 2 & 12,5 & 79 & 50,3 \\
\hline Incorrecta (otro valor) & 42 & 29,8 & 11 & 68,8 & 53 & 33,8 \\
\hline Otra respuesta & 3 & 2,1 & & & 3 & 1,9 \\
\hline No responde & 19 & 13,5 & 3 & 18,8 & 22 & 14 \\
\hline Total & 141 & & 16 & & 157 & \\
\hline
\end{tabular}

Fuente: Datos de la investigación.

Tabla 3 - Frecuencia y porcentaje de respuestas de los futuros profesores al ítem 3

\begin{tabular}{l|c|c}
\hline \multicolumn{1}{c|}{ Respuesta } & Frecuencia & $\mathbf{\%}$ \\
\hline Correcta: Pasar 1By 1N de Pablo a Miguel (Cajas iguales) & 117 & 74,5 \\
\hline Correcta: Pasar 1B y 2N de Miguel a Pablo (Cajas proporcionales) & 3 & 1,9 \\
\hline Incorrecta: No modifica las cajas (Cajas no proporcionales) & 14 & 8,9 \\
\hline Incorrecta: Otras respuestas & 20 & 12,7 \\
\hline No responde (en blanco) & 3 & 1,9 \\
\hline Total & 157 & \\
\hline
\end{tabular}

Fuente: Datos de la investigación.

En consecuencia, la primera parte de la evaluación sugiere un conocimiento probabilístico insuficiente en los participantes en el estudio, en concordancia con las investigaciones citadas en los antecedentes, por ejemplo, de Azcárate (1995) o Mohamed (2012).

\section{Resultados sobre Conocimiento Especializado del Contenido}

Para evaluar las respuestas a la pregunta 1 de la segunda parte del cuestionario se contó el número correcto e incorrecto de objetos matemáticos que cada grupo de participantes citó al describir el contenido matemático requerido en las soluciones correctas a los ítems. Casi todos los grupos identificaron correctamente algún objeto matemático utilizado para resolver la tarea (Tabla 
4), aunque el número dado fue pequeño, en especial en el primer ítem. Los más frecuentemente identificados fueron:

- Entre los conceptos: azar, casos favorables, casos posibles, esperanza matemática, experimento aleatorio, fracciones, números, números naturales, posibilidades, probabilidad, juego equitativo, proporción.

- Entre las propiedades: carácter aleatorio de las experiencias descritas en los ítems, proporcionalidad, regla de Laplace, suceso más o menos probable, equivalencia de fracciones, múltiplos, relación entre los factores y producto.

- Entre los procedimientos: procedimientos combinatorios para la enumeración de posibilidades, asignación de probabilidades, comparar casos posibles o favorables, operaciones aritméticas, regla de tres, simplificación de fracciones.

Tabla 4 - Número de objetos matemáticos identificados por los grupos de futuros profesores en las tareas y porcentaje de grupos que identifican ese número

\begin{tabular}{c|c|c|c|c|c|c}
\hline \multirow{2}{*}{ Número } & \multicolumn{2}{|c|}{ Ítem 1 } & \multicolumn{2}{c|}{ Ítem 2 } & \multicolumn{2}{c}{ Ítem 3 } \\
\cline { 2 - 7 } & Frecuencia & $\mathbf{\%}$ & Frecuencia & $\mathbf{\%}$ & Frecuencia & $\mathbf{\%}$ \\
\hline $\mathbf{0}$ & 8 & 20 & 8 & 20 & 7 & 17,5 \\
\hline $\mathbf{1}$ & 3 & 7,5 & 2 & 5 & 4 & 10 \\
\hline $\mathbf{2}$ & 10 & 25 & 9 & 22,5 & 4 & 10 \\
\hline $\mathbf{3}$ & 11 & 27,5 & 3 & 7,5 & 10 & 25 \\
\hline $\mathbf{4}$ & 4 & 10 & 4 & 10 & 2 & 5 \\
\hline $\mathbf{5}$ & 4 & 10 & 4 & 10 & 8 & 20 \\
\hline $\mathbf{6}$ o más & 0 & 0 & 10 & 25 & 5 & 12,5 \\
\hline Total & 40 & & 40 & & 40 & \\
\hline
\end{tabular}

Fuente: Datos de la investigación.

También se identificaron objetos que no aparecen en las actividades, tales como asignación frecuencial de la probabilidad o media, y se hizo referencia a objetos no matemáticos (como el sentido común). No llegaron a nombrarse el experimento aleatorio compuesto, espacio muestral compuesto, o la independencia.

Nuestros resultados son mejores que los de Ortiz, Batanero y Contreras (2012) y Mohamed (2012), quienes proponen la misma tarea (identificar el contenido matemático requerido en la solución correcta) en otros problemas de probabilidad. El número de objetos correctamente identificados en nuestro estudio fue algo mayor que el obtenido por estos autores y apenas aparecen objetos no necesitados en la solución del problema, al contrario que en dichos estudios. Puesto que los participantes en las investigaciones citadas no discutieron previamente las soluciones al problema analizado, ni realizaron actividades de simulación, deducimos que el proceso formativo seguido con los participantes 
en el estudio pudo desarrollar algo su conocimiento especializado del contenido. En todo caso, esta actividad es difícil y requiere más práctica por parte de los profesores en formación, de acuerdo a Chick y Pierce (2008), que también informan de fallos en la identificación de los conceptos latentes en una situación didáctica propuesta a 27 profesores de Educación Primaria en formación.

\section{Resultados sobre el Conocimiento del Contenido y los Estudiantes}

\section{Identificación de respuestas correctas e incorrectas}

En otras dos preguntas de la segunda parte del cuestionario se pidió a los participantes evaluar las respuestas de alumnos ficticios de Educación Primaria e indicar las causas de sus dificultades. La Tabla 5 resume sus valoraciones para cada respuesta.

Tabla 5 - Frecuencia y porcentaje de equipos que evalúan correctamente las respuestas de alumnos ficticios

\begin{tabular}{|c|c|c|c|c|c|c|}
\hline & \multirow{2}{*}{$\begin{array}{l}\text { Niño } \\
\text { ficticio }\end{array}$} & \multirow{2}{*}{ Tipo de error } & \multicolumn{2}{|c|}{ Evaluación idónea } & \multicolumn{2}{|c|}{ Razón satisfactoria } \\
\hline & & & Frecuencia & $\%$ & Frecuencia & $\%$ \\
\hline \multirow{5}{*}{ 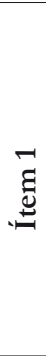 } & A1 & $\begin{array}{l}\text { Incorrecta: No se puede predecir, pero si } \\
\text { dar la probabilidad }\end{array}$ & 33 & 82,5 & 26 & 65,0 \\
\hline & A2 & $\begin{array}{l}\text { Incorrecta: No identifica el suceso más } \\
\text { probable; no enumera }\end{array}$ & 31 & 77,5 & 13 & 32,5 \\
\hline & A3 & $\begin{array}{l}\text { Incorrecta: enumeración incompleta; no } \\
\text { identifica el suceso menos probable }\end{array}$ & 18 & 45,0 & 13 & 32,5 \\
\hline & A4 & $\begin{array}{l}\text { P. correcta: Identifica el suceso más } \\
\text { probable, pero no el menos probable }\end{array}$ & 35 & 87,5 & 11 & 27,5 \\
\hline & A5 & Correcta & 36 & 90,0 & \multicolumn{2}{|c|}{ No se pide justificar } \\
\hline \multirow{5}{*}{ ثِّ } & B1 & $\begin{array}{l}\text { Incorrecta: Comparaciones aditivas; } \\
\text { premio incorrecto }\end{array}$ & 38 & 95,0 & 26 & 65,0 \\
\hline & B2 & $\begin{array}{l}\text { Incorrecta: Premio incorrecto; falta de } \\
\text { proporcionalidad }\end{array}$ & 37 & 92,5 & 13 & 32,5 \\
\hline & B3 & $\begin{array}{l}\text { Parcialmente correcta. Añade condición } \\
\text { innecesaria }\end{array}$ & 30 & 75,0 & 11 & 27,5 \\
\hline & B4 & $\begin{array}{l}\text { Incorrecta: No comprende el concepto de } \\
\text { juego equitativo }\end{array}$ & 25 & 62,5 & 14 & 35,0 \\
\hline & B5 & Correcta & 34 & 85,0 & \multicolumn{2}{|c|}{ No se pide justificar } \\
\hline \multirow{4}{*}{$\stackrel{n}{\mathscr{E}}$} & $\mathrm{C} 1$ & Incorrecta: Varía el número total de bolas & 37 & 92,5 & 17 & 42,5 \\
\hline & $\mathrm{C} 2$ & $\begin{array}{l}\text { Incorrecta: Estrategia univariada: solo } \\
\text { considera casos desfavorables }\end{array}$ & 35 & 87,5 & 18 & 45,0 \\
\hline & C3 & Correcta & 35 & 87,5 & \multicolumn{2}{|c|}{ No se pide justificar } \\
\hline & $\mathrm{C} 4$ & $\begin{array}{l}\text { Incorrecta: Confunde el nombre de los } \\
\text { niños; fallo razonamiento proporcional }\end{array}$ & 24 & 60,0 & 11 & 27,5 \\
\hline
\end{tabular}

Fuente: Datos de la investigación. 
Hemos considerado que la evaluación realizada es correcta si el profesor en formación identifica adecuadamente una respuesta como correcta, parcialmente correcta o incorrecta. Podemos observar que la mayoría de los grupos realiza una evaluación adecuada de la corrección o incorrección de cada respuesta, con resultados similares a los de Mohamed (2012), que obtuvo entre el $45 \%$ y el 97\% de respuestas de niños ficticios evaluadas correctamente.

El mayor éxito en la tarea de evaluación, respecto a la resolución previa del problema, puede ser debido a que los participantes trabajaron en parejas o grupos (en tanto resolvieron individualmente los problemas). El único caso algo más difícil de identificar fue el razonamiento de A3 (enumeración combinatoria incompleta), posiblemente porque también los profesores en formación tienen dificultades de enumeración.

\section{Justificación de las causas del error en el ítem 1 (sesgo de equiprobabilidad)}

Asimismo se categorizaron las justificaciones dadas para identificar las respuestas incorrectas. A continuación se incluyen las categorías empleadas en el ítem 1 , junto con un ejemplo de cada una de ellas:

E1. Sesgo de equiprobabilidad (LECOUTRE, 1992). Estos equipos indican que el niño ficticio no reconoce que los sucesos tienen probabilidades diferentes porque asocia el azar con equiprobabilidad. En el ejemplo siguiente, el grupo, además, reconoce que aunque los sucesos aislados son impredecibles se puede calcular la probabilidad de cada suceso (BATANERO; SERRANO, 1999):

El alumno A1 se ha dejado llevar por la hipótesis de equiprobabilidad. Esto es erróneo, porque, aunque es un juego de azar y no se puede predecir con certeza (adivinar) el resultado, sí podemos calcular las probabilidades para cada uno de los resultados (G37).

E2. Razonamiento combinatorio insuficiente. Se indica que el niño ficticio se equivoca al calcular probabilidades debido a una enumeración incompleta de casos favorables o posibles, reconociendo la importancia del razonamiento combinatorio para resolver el problema. En ocasiones se completa una enumeración correcta:

A4 responde correctamente al indicar que "es más difícil obtener tres veces el 5, para los otros resultados tienes más casos y para este solo 1". Pero para que la respuesta fuese perfecta, podría haber aclarado el alumno que para la opción 1 (un 5, un 2, y un 3) existen seis casos posibles y que para la opción 2 (dos veces el 5 y una el 3) existen 3 casos (G21).

E3. Ratifican la parte correcta e indican la respuesta a la parte incorrecta. No se mencionan posibles intuiciones incorrectas o estrategias que llevan al error, sino 
se completa la respuesta para que sea correcta:

Para la respuesta de A3: Sí que es más fácil obtener un 5, 2 y 3 pero porque tiene 6 casos diferentes, no 3 (G27).

E4. Explicación insuficiente. Estos equipos indican que el niño ficticio falló porque no calcula el número de casos favorables a cada posible respuesta; pero dan una explicación insuficiente del error cometido:

Para la respuesta de A2: Piensa que es más probable que salgan números diferentes (G11).

En la Tabla 6 se recogen las justificaciones de los grupos (recordamos que no era preciso explicar la respuesta correcta A5). Consideramos aceptables los argumentos basados en el sesgo de equiprobabilidad (E1), razonamiento combinatorio insuficiente (E2), o corrección de la parte incorrecta (E3) para el alumno A1 y estas dos últimas justificaciones (E2 y E3) para A2, A3 y A4. Vemos que una parte importante de los grupos es capaz de explicar las respuestas incorrectas, pero el porcentaje es mucho menor que los que las identifican adecuadamente (pregunta anterior).

Tabla 6 - Frecuencias (y porcentaje) de justificaciones de respuestas incorrectas en el ítem 1

\begin{tabular}{l|c|c|c|c|c|c|c|c}
\hline \multirow{2}{*}{ Explicación } & \multicolumn{2}{c|}{$\begin{array}{c}\text { A1 } \\
\text { (incorrecto) }\end{array}$} & \multicolumn{2}{c|}{$\begin{array}{c}\text { A2 } \\
\text { (incorrecto) }\end{array}$} & \multicolumn{2}{c|}{$\begin{array}{c}\text { A3 } \\
\text { (incorrecto) }\end{array}$} & \multicolumn{2}{c}{$\begin{array}{c}\text { A4 } \\
\text { (P. correcto) }\end{array}$} \\
\cline { 2 - 9 } & Frecuencia & $\%$ & Frecuencia & $\%$ & Frecuencia & \% & Frecuencia & $\%$ \\
\hline $\begin{array}{l}\text { E1. Sesgo de } \\
\text { equiprobabilidad }\end{array}$ & 19 & 47,5 & 8 & 20,0 & 1 & 2,5 & & \\
\hline $\begin{array}{l}\text { E2. Razonamiento } \\
\text { combinatorio insuficiente }\end{array}$ & 7 & 17,5 & 5 & 12,5 & 12 & 30,0 & 11 & 27,5 \\
\hline $\begin{array}{l}\text { E3. Ratifican lo correcto o } \\
\text { corrigen la parte incorrecta }\end{array}$ & 3 & 7,5 & 16 & 40,0 & 10 & 25,0 & 1 & 2,5 \\
\hline $\begin{array}{l}\text { E4. Explicaciones } \\
\text { insuficientes }\end{array}$ & 7 & 17,5 & 9 & 22,5 & 5 & 12,5 & 2 & 5,0 \\
\hline No justifica & 4 & 10,0 & 2 & 5,0 & 12 & 30,0 & 26 & 65,0 \\
\hline Total & 40 & & 40 & & 40 & & 40 & \\
\hline
\end{tabular}

Fuente: Datos de la investigación.

\section{Justificación de las causas del error en el ítem 2 (juego equitativo)}

En este caso se han considerado los siguientes tipos de justificaciones:

E1. Error en el cálculo de probabilidades. Algunos equipos indican que el alumno se equivocó en el cálculo de las probabilidades, dando la probabilidad correcta o bien el número casos favorables:

B2 se ha equivocado haciendo las probabilidades de los casos porque Luis tiene 9 probabilidades (casos favorables) mientras que Miguel tiene 27 probabilidades (G040). 
E2. Error en el cálculo del premio. Estos equipos indican que el alumno calcula correctamente las probabilidades pero se equivoca al asignar la ganancia para Luis:

Para el alumno B2: Se ha equivocado porque ha relacionado el 6 con el número de caras que tiene un dado (G18).

E3. Condición innecesaria. Otros grupos identifican la condición innecesaria introducida por B3, indicando que el alumno muestra una concepción errónea de juego equitativo, similar a la descrita en niños por Cañizares (1997):

Toma dos medidas para equilibrar el juego que individualmente estarían bien, pero las dos juntas benefician mucho a Luis (G06).

E4. Dan la respuesta correcta. Algunos equipos escriben cuál sería la respuesta correcta a la parte que está incorrecta, pero no mencionan posibles intuiciones incorrectas o estrategias que llevan al error:

Para el alumno B2: Debe de ganar $3 €$ en vez de $6 €$ como dice (G09).

E5. Otros argumentos. Otros argumentos minoritarios indican que la respuesta del alumno ficticio es incompleta, hacen referencia a uso de lenguaje inapropiado o bajo nivel de reflexión, o bien que el alumno no entiende el problema.

En la Tabla 7 se resumen los argumentos al evaluar alumnos ficticios en el ítem 2, donde cabe recordar que casi la mitad de los participantes calificaron las respuestas de $\mathrm{B} 3$ como correcta, por lo cual no trataron de justificar el posible error.

Tabla 7 - Frecuencias (y porcentaje) de justificaciones de respuestas incorrectas en el ítem 2

\begin{tabular}{l|c|c|c|c|c|c|c|c}
\hline \multicolumn{1}{c|}{ Explicación } & \multicolumn{2}{c|}{$\begin{array}{c}\text { B1 } \\
\text { (incorrecto) }\end{array}$} & \multicolumn{2}{c|}{$\begin{array}{c}\text { B2 } \\
\text { (incorrecto) }\end{array}$} & \multicolumn{2}{c|}{$\begin{array}{c}\text { B3 } \\
\text { (P. correcto) }\end{array}$} & \multicolumn{2}{c}{$\begin{array}{c}\text { B4 } \\
\text { (incorrecto) }\end{array}$} \\
\hline & Frecuencia & $\%$ & Frecuencia & $\%$ & Frecuencia & $\%$ & Frecuencia & $\%$ \\
\hline $\begin{array}{l}\text { E1. Error al calcular } \\
\text { probabilidades }\end{array}$ & 28 & 70,0 & 6 & 15,0 & 2 & 5,0 & & \\
\hline $\begin{array}{l}\text { E2. Error en cálculo } \\
\text { del premio }\end{array}$ & 2 & 5,0 & 8 & 20,0 & & & 14 & 35,0 \\
\hline $\begin{array}{l}\text { E3. Condición } \\
\text { innecesaria }\end{array}$ & & & & & 11 & 27,5 & & \\
\hline $\begin{array}{l}\text { E4. Indican respuesta } \\
\text { correcta }\end{array}$ & 4 & 10,0 & 9 & 22,5 & & & 2 & 5,0 \\
\hline E5. Otras explicaciones & 3 & 7,5 & 12 & 30,0 & 6 & 15,0 & 11 & 27,5 \\
\hline No justifica & 3 & 7,5 & 5 & 12,5 & 21 & 52,5 & 13 & 32,5 \\
\hline Total & 40 & & 40 & & 40 & & 40 & \\
\hline
\end{tabular}

Fuente: Datos de la investigación. 
El argumento E1 (error en cálculo de probabilidades) es aceptable para el error de B1; E1 o E2 (error en cálculo del premio) para B2; E3 (condición innecesaria) para B3, y E2 para B4. Observamos que muchos grupos dan una explicación aceptable del error, en especial para los alumnos B1 y B4, y también se detecta la parte incorrecta en la respuesta de B3, es decir, la imposición de una condición innecesaria en el juego (E3).

Estos resultados son mejores que los de Mohamed (2012), pues pocos grupos en su investigación detectaron las fuentes de los errores de los niños en un problema sobre juego equitativo: el fallo en el cálculo de probabilidades fue identificado en una de las respuestas por el 39\% de los grupos de participantes, y el error consistente en añadir una condición innecesaria fue identificado por 13\%; solo algún grupo aislado pudo identificar otros errores. Adicionalmente, la tasa de no respuesta en el análisis de los argumentos fue más alta que la nuestra.

\section{Justificación de las causas del error en el ítem 3 (comparación de probabilidades)}

Las justificaciones utilizadas en este caso son las siguientes:

E1. Excede el total. Se indica que el niño ficticio propone una solución incorrecta debido a que no respeta las condiciones del enunciado:

En parte es correcta porque añadiendo dos bolas de cada a la caja de Miguel tendrían la misma probabilidad, pero el enunciado pide pasar bolas de uno a otro niño; no añadir. Por lo que el niño ha confundido el enunciado (G18).

E2. Solución incorrecta. No indican posibles intuiciones incorrectas o estrategias que llevan al error; sólo señalan que el niño hace algo incorrecto:

No se puede; no está claro el origen de las bolas ya que no las quita de la caja de Pablo (G34).

E3. El alumno utiliza una estrategia univariada. Se basa en que el niño tiene en cuenta sólo los casos desfavorables o favorables:

El niño no ha tenido en cuenta las bolas negras (G08).

E4. Distinta probabilidad (o proporción): Algunos equipos tienen en cuenta que no hay proporcionalidad en las cajas, o que la probabilidad de extraer la bola negra es diferente para Miguel y para Pablo. Por ejemplo:

Para la respuesta del alumno C2: 4/7 y 4/5 no está bien la proporción (G26).

E5. Igualdad de las cajas. Estos equipos comparan la modificación de cajas, dada por el alumno ficticio, con una solución correcta que conlleva a tener dos cajas iguales. En ocasiones se indica también una confusión de interpretación del enunciado: 
Para la respuesta del alumno C4: si es cierto que hay que pasar ese número de bolas, es decir el planteamiento está bien, pero hay que pasarlas de la caja de Pablo a la de Miguel y no al contrario (G11).

E6. Proceso incompleto. Estos equipos atribuyen el fallo del alumno a una omisión de una parte del procedimiento o de su explicación, teniendo en cuenta que la solución dada por el alumno ficticio difiere de la respuesta correcta en un paso:

Para la respuesta del alumno C2: ¡Olvida hacer los cambios necesarios en las bolas negras! Nosotras consideramos que puede ser debido simplemente a que olvidó resolver la segunda parte del problema, puesto que se queda satisfecho con resolver la primera parte (G19).

Otros argumentos minoritarios se refieren a errores de cálculo, considerar ilógico el planteamiento, fijarse en la diferencia entre favorables y desfavorables o explicaciones inconsistentes.

Las justificaciones presentadas en la Tabla 8 muestran una argumentación insuficiente; en general, hay menos explicaciones probabilísticas (E1, E3, E4) que de tipo normativo (E2, E5, E6). El mejor desempeño se observa en la explicación del error por exceso en el total de bolas (C1) o intercambio en el nombre de los niños (C4), siendo difícil explicar el error por usar una estrategia univariada (C2).

Sin embargo los resultados son mejores que los de Mohamed (2012), quien identificó solo tres tipos de explicación en sus estudiantes: dar la respuesta correcta (en nuestro caso está dividida en tres categorías), y no tener en cuenta la proporción o probabilidad (que nosotros hemos reunido en una sola). Adicionalmente, la tasa de no respuesta fue más alta que la nuestra.

Tabla 8 - Frecuencias (y porcentaje) de justificaciones de respuestas incorrectas en el ítem 3

\begin{tabular}{l|c|c|c|c|c|c}
\hline \multirow{2}{*}{ Explicación } & \multicolumn{2}{c|}{ C1 (incorrecto) } & \multicolumn{2}{c|}{ C2 (incorrecto) } & \multicolumn{2}{c}{ C4 (incorrecto) } \\
\cline { 2 - 7 } & Frecuencia & \% & Frecuencia & \% & Frecuencia & \% \\
\hline E1. Excede el total & 16 & 40,0 & & & & \\
\hline E2. No se puede hacer & 14 & 35,0 & & & & \\
\hline E3. Estrategia univariada & & & 6 & 15,0 & & \\
\hline E4. Distinta probabilidad & 1 & 2,5 & 12 & 30,0 & 7 & 17,5 \\
\hline E5. Cajas iguales & 1 & 2,5 & 9 & 22,5 & 9 & 22,5 \\
\hline E6. Proceso incompleto & 1 & 2,5 & 6 & 15,0 & 1 & 2,5 \\
\hline E7. Otras explicaciones & 4 & 10,0 & 3 & 7,5 & 11 & 27,5 \\
\hline No justifica & 3 & 7,5 & 4 & 10,0 & 12 & 30,0 \\
\hline Total & 40 & & 40 & & 40 & \\
\hline
\end{tabular}

Fuente: Datos de la investigación. 


\section{Discusión e implicaciones para la formación de profesores}

En este trabajo se propusieron una serie de tareas de probabilidad elemental con el fin de evaluar y desarrollar algunos componentes del conocimiento matemático para la enseñanæa (BALL; LUBIENSKI; MEWBORN, 2001; HILL; BALL; SCHILLING, 2008) en un grupo de profesores de Educación Primaria en formación. Las principales conclusiones obtenidas respecto a los componentes de dicho conocimiento en esta muestra de profesores se describen a continuación.

En cuanto al Conocimiento Común de la probabilidad, los datos recogidos de la primera parte del cuestionario muestran un pobre conocimiento inicial de la probabilidad por parte de los futuros profesores, observándose la presencia del sesgo de equiprobabilidad en una amplia mayoría. Sólo la mitad de la muestra fue capaz de calcular el valor esperado de ganancia para convertir un juego no equitativo en equitativo y muy pocos logran formar dos urnas con la misma probabilidad pero proporcionales (en lugar de con idéntica composición). La identificación de las respuestas correctas e incorrectas de niños ficticios a los mismos ítems en la segunda parte del cuestionario, indica sin embargo que su conocimiento mejora, lo que atribuimos al aprendizaje logrado por parte de los profesores en formación, mediante la actividad propuesta.

En concordancia con estudios previos con profesores en formación (CHICK; PIERCE, 2008; ORTIZ; BATANERO; CONTRERAS, 2012; MOHAMED, 2012) nuestros resultados indican pobre Conocimiento Especializado del Contenido, y falta de capacidad para identificar los objetos matemáticos en una tarea, incluso cuando los futuros profesores utilizaron dichos objetos al resolver correctamente los problemas planteados. De todos modos los resultados son algo mejores que los obtenidos en los trabajos citados, pensamos que como consecuencia del aprendizaje logrado en la segunda sesión. Será importante buscar formas alternativas de desarrollar este conocimiento especializado, pues su carencia podría dificultar algunas de las actividades que realiza el profesor. Entre estas actividades citamos proponer problemas adecuados para el aprendizaje de un tema, variar el contenido o formato de una tarea para hacerla más sencilla o más difícil, o identificar los conocimientos matemáticos que utiliza un alumno en sus respuestas a los ítems de evaluación (WATSON, 2001; STOHL, 2005).

Finalmente, en relación al Conocimiento del Contenido y los Estudiantes, los resultados también son mejores que los informados en estudios previos; especialmente la capacidad de justificación de las respuestas incorrectas. Un alto porcentaje de los participantes en nuestro estudio fueron capaces de dar razones válidas para los errores en las respuestas de niños ficticios a los ítems; mostrando mejores resultados incluso que algunos estudios con profesores en ejercicio (WATSON, 2001; STOHL, 2005) o en formación (CARTER, 2008; 
MOHAMED, 2012). Los razonamientos erróneos mejor identificados fueron el sesgo de equiprobabilidad y razonamiento combinatorio insuficiente (en el ítem 1); error en el cálculo de la probabilidad, error en el establecimiento del premio o imposición de una condición innecesaria en el ítem 2, y exceso en el total de casos posibles o cambio en las condiciones del problema en el ítem 3.

Pensamos que el ciclo formativo seguido ha ayudado a mejorar el conocimiento de los futuros profesores. En este ciclo, una vez resuelta la primera parte del cuestionario en forma individual, se debaten colectivamente estas respuestas, diferenciando las correctas e incorrectas, con apoyo de actividades de simulación y de enumeración del espacio muestral, dejando siempre que sean los propios participantes quienes lleguen a reconocer sus errores previos. Posteriormente, trabajando en pequeños grupos, los futuros profesores realizan actividades de evaluación de respuestas de alumnos ficticios, a los mismos ítems y justificando las razones por las que consideran cada respuesta correcta o incorrecta, razonando asimismo, sobre el contenido matemático requerido para la solución correcta de cada tarea.

El haber discutido colectivamente sus propias respuestas incorrectas a la primera parte de la tarea, como parte de este ciclo formativo, sirvió, en la segunda parte para discriminar con facilidad las respuestas correctas e incorrectas de los alumnos ficticios e incluso en una proporción importante justificar las causas de sus errores. Por ello deducimos que el avance en su conocimiento común del contenido ayudó a desarrollar su conocimiento del contenido y los estudiantes.

Una implicación de interés para los formadores de profesores de Educación Primaria es la necesidad de reforzar su formación para la enseñanza de la probabilidad, en sus distintos componentes. La probabilidad es un tema incluido muy recientemente en el currículo de Educación Primaria; por ello algunos de los futuros profesores no la estudiaron durante dicho periodo escolar, como fue el caso de la mayoría de participantes en nuestro estudio, lo que explica los pobres resultados en la primera parte del cuestionario.

En consecuencia, en el caso de que la formación probabilística de los futuros profesores en la educación secundaria y en el bachillerato fuese deficiente, deberá ser una tarea de las facultades de educación el completarla en forma adecuada. Aunque nuestra experiencia ha sido limitada a pocas sesiones de trabajo, los resultados en la segunda parte del cuestionario también muestran que es posible mejorar el conocimiento de los profesores, aún con un tiempo limitado de enseñanza.

Respecto a la metodología, para llevar a cabo esta formación, se sugiere proponer a los futuros profesores una muestra de situaciones experimentales contextualizadas en su futura labor profesional, como se ha realizado en este 
trabajo, donde hemos propuesto situaciones de evaluación y de identificación del contenido matemático en tareas escolares, junto con actividades de simulación que pueden utilizar con sus futuros estudiantes.

La resolución previa de los problemas y discusión colectiva de las soluciones apoyada en la simulación proporcionó también a los futuros profesores un modelo sobre la metodología de enseñanza de la probabilidad; donde conviene confrontar a los estudiantes con sus propios sesgos e intuiciones incorrectas, darles oportunidad de debatir estas ideas con sus compañeros y proporcionarles, por medio de la simulación, una mayor experiencia con los fenómenos aleatorios que les lleven a corregir y rechazar estas intuiciones incorrectas.

Para preparar a los profesores en la componente didáctica, serán de gran ayuda situaciones relacionadas con la docencia, como las usadas en este trabajo, pues de acuerdo a Llinares (2009) estas tareas tienen en cuenta los contextos en que el futuro maestro ha de aplicar su conocimiento en la práctica de enseñar matemáticas.

Estas tareas cumplen también algunos requisitos señalados por Suzuka et al. (2009) para ser útiles en el desarrollo del conocimiento matemático para la enseñanza, pues proporcionan una oportunidad de explicitar y desarrollar las ideas matemáticas centrales en el currículo, y permiten establecer conexiones entre ideas matemáticas, así como entre múltiples representaciones y métodos de solución. También permiten la práctica con actividades centrales a la enseñanza, como es la evaluación de los estudiantes y la identificación de sus formas de razonamiento y sus errores. Por supuesto sería necesario dedicar un mayor tiempo a esta formación inicial, completando con otros contenidos de probabilidad y su didáctica los tratados en esta investigación, donde tuvimos la imposición de la limitación del tiempo.

Resaltamos también la necesidad de continuar la investigación sobre otros componentes del conocimiento del profesor en el campo de la probabilidad, como paso necesario para desarrollar propuestas de enseñanza que contribuyan a mejorar la formación de los profesores.

Agradecimientos: Proyecto EDU2010-14947 (MCINN-FEDER) y grupo FQM126 (Junta de Andalucía).

\section{Referencias}

AZCÁRATE, P. El conocimiento profesional de los profesores sobre las nociones de aleatoriedad y probabilidad: su estudio en el caso de la educación primaria. 1995. 918 f. Tesis (Doctorado en Educación) - Departamento de Didáctica, Universidad de Cádiz, Cádiz, 1995. 
BALL, D. L.; LUBIENSKI, S. T.; MEWBORN, D. S. Research on teaching mathematics: the unsolved problem of teachers' mathematical knowledge. In: RICHARDSON, V. Handbook of research on teaching. Washington: American Educational Research Association, 2001. p. 433-456.

BALL, D. L.; THAMES, M. H.; PHELPS, G. Content knowledge for teaching: what makes it special? Journal of Teacher Education, Pennsylvania, v. 59, n. 5, p. 389-407, Nov./Dec. 2008. DOI: $10.1177 / 0022487108324554$

BATANERO, C.; ARTEAGA, P.; SERRANO, L.; RUIZ, B. Prospective primary school teachers' perception of randomness. In: CHERNOFF, E. J.; SRIRAMAN, B. Probabilistic thinking. Heidelberg: Springer, 2014. p. 345-366.

BATANERO, C.; CAÑIZARES, M. J.; GODINO, J. Simulation as a tool to train preservice school teachers. In: ICMI FIRST AFRICAN REGIONAL CONFERENCE, 2005, Johannesburgo. Proceedings... Johannesburgo: International Commission on Mathematical Instruction. 2005. CD-ROM.

BATANERO, C.; SERRANO, L. The meaning of randomness for secondary school students. Journal for Research in Mathematics Education, Virginia, v. 30, n. 5, p. 558-567, Nov. 1999. DOI: $10.2307 / 749774$

CAÑIZARES, M. J. Influencia del razonamiento proporcional y combinatorio y de creencias subjetivas en las intuiciones probabilísticas primarias. 1997. 192 f. Tesis (Doctorado en Didáctica de la Matemática) - Departamento de Didáctica de la Matemática, Universidad de Granada, Granada, 1997. Disponible en: <http://www. ugr.es/local/ batanero>. Acceso en: 4 nov. 2013.

CARTER, T. A. Preservice teacher knowledge and understanding of probability and statistics. In: KULM, G. Teacher knowledge and practice in middle grades mathematics. Rotterdam: Sense Publishers, 2008. p. 19-43.

COMMON CORE STANDARDS INITIATIVE, CCSI. Standards for mathematical practice. 2010. Disponible en: <http://www.corestandards.org/>. Acceso en: 4 nov. 2013.

CONTRERAS, J. M. Evaluación de conocimientos y recursos didácticos en la formación de profesores sobre probabilidad condicional. 2011. 250 f. Tesis (Doctorado en Didáctica de la Matemática) - Departamento de Didáctica de la Matemática. Universidad de Granada, Granada, 2011. Disponible en: <http://www. ugr.es/local/batanero>. Acceso en: 18 ago. 2013.

CHICK, H. L.; PIERCE, R. U. Teaching statistics at the primary school level: beliefs, affordances, and pedagogical content knowledge. In: JOINT ICMI/IASE STUDY STATISTICS IN SCHOOL MATHEMATICS. CHALLENGES FOR TEACHING AND TEACHER EDUCATION, 2008, Monterrey. Proceedings... Monterrey: ICMI and IASE, 2008. p. 1-8. Disponible en: <http://www.ugr.es/ icmi/iase_study/>. Acceso en: 5 sep. 2012.

EVEN, R.; BALL, D. The professional education and development of teachers of mathematics. ICMI Study 15. New York: Springer, 2009.

FALK, R.; WILKENING, F. Children's construction of fair chances: adjusting probabilities. Developmental Psychology, Michigan, v. 34, n. 6, p. 1340-1357, Nov. 1998. DOI: 10.1037//0012-1649.34.6.1340

GODINO, J. D. Categorías de análisis de los conocimientos del profesor de matemáticas. 
UNIÓN. Revista iberoamericana de Educación Matemática, Buenos Aires, n. 20, p. 13-31, dic. 2009.

HILL, H.; BALL, D. L.; SCHILLING, S. Unpacking pedagogical content knowledge: conceptualizing and measuring teachers' topic-specific knowledge of students. Journal for Research in Mathematics Education, Virginia, v. 39, n. 4, p. 372-400, Jul. 2008.

LECOUTRE, M. P. Cognitive models and problem spaces in "purely random" situations. Educational Studies in Mathematics, Dordrecht, v. 23, p. 557-568, Dec. 1992. DOI: 10.1007/BF00540060

LLINARES, S. Competencias docentes del maestro en la docencia en matemáticas y el diseño de programas de formación. UNO. Revista de Didáctica de las Matemáticas, Barcelona. v. 51, p. 92-101, abr. 2009.

LLINARES, S.; KRAINER, K. Mathematics (student) teachers and teacher educators as learners. In: GUTIERREZ, A.; BOERO, P. Handbook of research on the psychology of mathematics education: past, present and future. Rotterdam/Taipei: Sense Publishers, 2006. p. 429-460.

MEC. Real Decreto 1513/2006, de 7 de diciembre, por el que se establecen las enseñanzas mínimas de la Educación Primaria. España: Ministerio de Educación y Cultura, 2006.

MOHAMED, N. Evaluación del conocimiento de los futuros profesores de Educación Primaria sobre probabilidad. 2012. 286 f. Tesis (Doctorado en Didáctica de la Matemática) - Departamento de Didáctica de la Matemática. Universidad de Granada, Granada, 2012. Disponible en: <http://www.ugr.es/local/batanero>. Acceso en: 4 nov. 2013.

NATIONAL COUNCIL OF TEACHERS OF MATHEMATICS, NCTM. Principles and standards for school mathematics. Reston, Virginia: National Council of Teachers of Mathematics, 2000.

ORTIZ, J.; BATANERO, C.; CONTRERAS, C. Conocimiento de profesores en formación sobre la idea de juego equitativo. Revista Latino Americana de Matemática Educativa, México, v. 15, n. 1, p. 63-91, marzo 2012.

PIAGET, J.; INHELDER, B. La genése de l'idée de hasard chez l'enfant. Paris: Presses Universitaires de France, 1951.

PRODROMOU, T. Connecting experimental probability and theoretical probability. ZDM, The International Journal of Mathematics Education, Hamburgo, v. 44, p. 855-868, Nov. 2012. DOI: $10.1007 /$ s11858-012-0469-z

SERRANO, L. Significados institucionales y personales de objetos matemáticos ligados a la aproximación frecuencial de la enseñanza de la probabilidad. 1996. 223 f. Tesis (Doctorado en Didáctica de la Matemática) - Departamento de Didáctica de la Matemática. Universidad de Granada, Granada, 1996. Disponible en: < http://www.ugr.es/ local/batanero>. Acceso en: 4 nov. 2013.

SHULMAN, L. S. Paradigm and research programs in the study of teaching: a contemporary perspective. In: WITROCK, M. C. Handbook of research on teaching. New York: Macmillan, 1986. p. 3-36. 
SMITH, T.; HJALMARSON, M. Eliciting and developing teachers' conceptions of random processes in a probability and statistics course. Mathematical Thinking and Learning, Queensland, v. 15, n. 1, p. 58-82, Jan. 2013. DOI: 10.1080/10986065.2013.738378

STOHL, H. Facilitating students' problem solving in a technological context: Prospective teachers' learning trajectory in technological contexts. Journal of Mathematics Teacher Education, Dordrecht, v. 8, n. 3, p. 223-254, June 2005. DOI: 10.1007/s10857-005-2618-6

SUZUKA, K.; SLEEP, L.; BALL, D. L.; BASS, H.; LEWIS, J. M.; THAMES, M. H. Designing and using tasks to teach mathematical knowledge for teaching. In: MEWBORN, D.; LEE, H. S. Inquiry into mathematics teacher education: monograph of the Association of Mathematics Teacher Education. San Diego: Association of Mathematics Teacher Education, 2009. p. 7-23.

WATSON, J. M. Profiling teachers' competence and confidence to teach particular mathematics topics: the case of chance and data. Journal of Mathematics Teacher Education, Dordrecht, v. 4, p. 305-337, Dec. 2001. DOI: 10.1023/A:1013383110860

WOOD, T. The international handbook of mathematics teacher education. Rotterdam: Sense Publishers, 2008.

Recebido em 17/11/2013

Aceito em 22/05/2014 